\title{
La dislipidemia en adultos y su tratamiento farmacológico
}

\author{
Dyslipidemia in adults and its \\ pharmacologic treatment
}

José B. Zapata Jaramillo

Turora: María C. Noguera Santamaría

\section{Resumen}

Las dislipidemias como condiciones patológicas cuyo único elemento común es una alteración del metabolismo de los lípidos, tienen una consecuente alteración de las concentraciones de colesterol y triglicéridos en sangre, que aumentan el riesgo de ateroesclerosis, cardiopatía e hipertensión. Esta revisión muestra los últimos estudios sobre la terapéutica médica con hipolipemiantes, además del uso correcto de estos, no solo teniendo en cuenta la dislipidemia a tratar, sino también la comorbilidad asociada y los beneficios concebidos con la correcta elección del fármaco. El uso de fármacos hipolipemiantes como las estatinas principalmente, fibratos y resinas fijadoras de colesterol, han demostrado un mayor beneficio en la reducción de las complicaciones de las diferentes enfermedades relacionadas en tolerancia y respuesta, con el fin de mejorar la condición del paciente.

Palabras clave: enfermedades cardiovasculares, HDL, LDL, VLDL, quilomicrones, obesidad, triglicéridos.

1 Estudiante de Medicina, Facultad de Ciencias de la Salud, Corporación Universitaria Remington, calle 51 \# 51-27, Medellín, Colombia.

2 Bacterióloga, Ph.D., docente Facultad de Ciencias de la Salud, Corporación Universitaria Remington, calle 51 \# 51-27, Medellín, Colombia.

\section{Abstract}

Dyslipidemias, a variety of disorders sharing the single common feature of impairment of lipid metabolism, are associated to a consequent alteration of cholesterol concentrations and triglycerides in blood, increasing risk for atherosclerosis, heart disease and hypertension. This review describes the most recent studies on medical 
therapy with lipid-lowering medications as well as their proper use according to the type of dyslipidemia to be treated, the associated comorbidity and the benefits derived from the correct choice of medications. The use of lipid-lowering drugs such as statins, fibrates and cholesterol-binding resins, have shown to have a greater benefit in reducing complications of the various diseases, regarding tolerance and response, leading to an improvement of the patient's condition.

Keywords: heart diseases, HDL, LDL, VLDL, chylomicrons, obesity, triglycerides.

\section{Introducción}

Existe un aumento en la frecuencia de las dislipidemias en el mundo y ha tomado dimensiones alarmantes por el aumento observado en la última década con una tendencia a progresión de la misma a futuro. Se ha encontrado que su aumento se relaciona directamente proporcional con la edad, y existe una mayor frecuencia en las mujeres teniendo en cuenta los valores de colesterol sérico encontrados. Con respecto a la hipercolesterolemia y dislipidemia mixta, se ha observado una mayor frecuencia en individuos con sobrepeso (obesos) por sus elevados valores de triglicéridos (Fernández Travieso, 2008a).

Esta revisión integra los diferentes estudios que se han publicado con respecto al uso y efectividad de los medicamento hipolipemiantes, y así facilita la labor del clínico en cuanto a la elección del fármaco por escoger, teniendo siempre en cuenta qué clase de dislipidemia se quiere tratar y las comorbilidades del paciente. También se pretende informar qué es lo último que se ha producido sobre el tema y las con- clusiones que se han arrojado en los últimos estudios. La presente revisión proporcionará recomendaciones para la buena práctica en cuanto a la medicación, que están basadas en la mejor evidencia clínica disponible y en las consideraciones adicionales relevantes en el contexto nacional.

\section{Dislipidemia}

Como trastorno en lípidos, la dislipidemia se caracteriza por el aumento en los niveles de colesterol (hipercolesterolemia), y de triglicéridos que es de mayor complicación (Travieso, J. C., 2008b).

Frecuentes en la medicina, son asistidas por alteraciones como la diabetes mellitus tipo I, gota y alcoholismo entre otras, complicando la condición del individuo aún más. Generalmente, se presenta un aumento para el riesgo de ateroesclerosis (Miguel Soca, 2009), el cual se mide de acuerdo con una clasificación clínica según la elevación del colesterol o los triglicéridos - primarias, genéticas o secundarias-y que se conoce como: hipertrigliceridemia aislada, hipercolesterolemia aislada, hiperlipidemia mixta y déficit de HDL aislado o hipoalfalipoproteinemia (Miguel Soca, 2009; Romero V, E., y Alemán, R., 2013); Martínez Hernández y Chávez Aguirre, 2007; López Pérez y Villar Novell, 2009).

Dentro de los estudios realizados se considera que la hipercolesterolemia aislada se puede atribuir a causas genéticas por herencia familiar y de tipo poligénico con múltiples factores involucrados, en donde existe mayor fuerza hacia factores ambientales como la adquisición de malos hábitos alimentarios y 
de sedentarismo (López Pérez y Villar Novell, 2009).

La aparición de hipertrigliceridemia aislada corresponde a defectos metabólicos de VLDL (very low-density lipoprotein; lipoproteínas de muy baja densidad), ya que los defectos severos se expresan como hiperlipidemia mixta debido al contenido significativo del colesterol de las VLDL. Otras causas genéticas reconocidas se atribuyen a factores hereditarios que se pueden encontrar asociados a causas patológicas secundarias, a la obesidad, diabetes y a la insuficiencia renal, entre otras. También en este caso se asocian factores poligénicos con una mala dieta con alto consumo de carbohidratos y alcohol (Martínez Hernández y Chávez Aguirre, 2007; Hooper L. et al, 2004). La hiperlipidemia mixta como combinación de las dos, genera complicaciones mayores con un componente genético de mayor complejidad que conllevaría a una posible diabetes (López Pérez y Villar Novell, 2009).

La generación de déficit aislado de HDL (High density lipoprotein; lipoproteínas de alta densidad) se ha relacionado como factor de riesgo de cardiopatía coronaria y se asocia a su disminución por causas metabólicas de las proteínas VLDL, a la existencia de entidades clínicas como las diabetes, obesidad y demás que han sido nombradas anteriormente. (Illnait Pérez, 2015; Miguel Soca, 2009; Martínez Hernández y Chávez Aguirre, 1997).

De acuerdo con los resultados de Euromonitor International, entre los años 1995 y 2012 se encontró un aumento de obesidad con cifras de interés que llegaron hasta un $26.54 \%$ de obesidad en Latinoamérica. En los últimos años, más del $50 \%$ de la población presenta sobrepeso (MachadoAlba et al., 2013). Otro hallazgo importante fue que en los últimos cinco años se presentó au- mento de sobrepeso en Centro y Suramérica de manera similar, exceptuando Ecuador, Argentina y Brasil (De los reyes, A., 2013).

En Estados Unidos, más de 97 millones de adultos son obesos y más de la mitad de la población presenta sobrepeso. El estudio NHANES (Encuesta Nacional de Examen de Salud y Nutrición) del Servicio de Salud Pública de Estados Unidos en el Departamento de Salud y Servicios Humanos de Estados Unidos, realizado en una población estadounidense entre los años 1960 y 1991, mostró que un tercio de la población mayor de 20 años presentaba sobrepeso y que había una clara tendencia al alza. Los resultados de análisis globales permitieron visualizar mejor el comportamiento de la dislipidemia de las poblaciones y su relación, resaltando el predominio de obesidad en la mujer. Se evidenció entonces un mayor riesgo cardiovascular en mujeres comparadas con los hombres (Luengo Fernández et al., 2005).

\section{Fisiopatología de la dislipidemia}

El mecanismo de circulación de los lípidos en el torrente sanguíneo se realiza en forma de lipoproteínas, moléculas que transportan los triglicéridos y ésteres de colesterol, los cuales se encuentran cubiertos por capa de fosfolípidos y algunas proteínas denominadas apoproteínas. Las lipoproteínas se encuentran como quilimicrones, VLDL (very low density lipoproteins o lipoproteínas de muy baja densidad), LDL (low density lipoprotein o de baja densidad) y HDL (high density lipoprotein o de alta densidad) (Morejón Reinoso, O. y Triana, M. E. (2015); Martínez Hernández Chávez Aguirre, 2007; Davies, 2007;). Las apoproteínas que se encuentran en dichas lipoproteínas tienen funciones protectoras para estas, ya que tienen receptores específicos. 
Las apoproteínas presentes en las lipoproteínas les confieren gran parte de sus características y funciones, ya que constituyen la porción de la molécula que es reconocida por ciertos receptores específicos. Su principal función es la de transportar los lípidos a la sangre (Hirsch, 2002; Sanhueza, J. et al, 2012). Teniendo en cuenta que los lípidos no se transportan fácilmente, se catabolizan en riñones y tejidos extrahepáticos (Xavier et al., 2005; Manrique, M. et al., 2009). El diagnóstico clínico se encuentra basado en la medición de niveles sérico de las lipoproteínas (Canalizo-Miranda et al., 2013).

De acuerdo con las guías «Adult Treatment Panel III Guidelines», se controvierte de manera importante la asociación de las hipertrigliceridemias con enfermedades como la arterioesclerosis y hacen asociaciones más cercanas con factores como la tensión arterial alta (Canalizo-Miranda et al., 2013).

\section{Implicaciones clínicas de la dislipidemia}

Las implicaciones clínicas se han venido relacionando con un incremento de la mortalidad vinculado con enfermedades secundarias, que se buscan reducir a través de estudios de prevención como el Nurses' Health Study y los de la Sociedad Americana contra el Cáncer, el Aerobic Center Longitudinal Study y el Finnish Heart Study; también de estudios como el realizado por el Informe del Examen Nacional de Salud y Nutrición de los Estados Unidos de Norteamérica (Troyo Barriga, 2004). Otros análisis soportan que es directamente proporcional el aumento de las diferentes unidades sérica medidas con la condición de sobrepeso que se presenta «NHANES (The National Health and Nutrition Examination Survey/La Encuesta Nacional de Examen de Salud y Nutrición)», au- menta la probabilidad de presentar un desorden metabólico (Martínez Hernández y Chávez Aguirre, 2007) o una predisposición cardiovascular (Fuster y Ruiz o, 2015). Actualmente, se asocia la arterioesclerosis como principal causa de mortalidad con consecuentes infartos de miocardio o cerebrales, aneurismas aórticos, etc. (Álvarez De Sotomayor y Herrera, 1999), el incremento de obesidad es causa de esto y a su vez factor de riesgo coronario (Luengo Fernández et al., 2005).

\section{Alteraciones en la estructura y la función cardiacas}

Las dislipidemias, ya sea por obesidad o como factor genético, son causa importante de insuficiencia cardiaca, al encontrar mecanismos fisiopatológicos relevantes para su diagnóstico como cambios hemodinámicos (aumento de precarga) y aumento de poscarga, efecto de la hipertensión asociada a hiperactividad simpática. Algunos otros cambios se deben a hipertrofia y aceleración de la arterioesclerosis presentada a nivel cardiaco. (Rubiés-Prat et al., 2003). Algunas enfermedades son ligadas a dislipidemias mas no son cardiovasculares, sin embargo, llegan a provocar fallo cardiaco teniendo como ejemplo el síndrome de apnea obstructiva del sueño (SAOS), (Luengo Fernández et al., 2005).

\section{Aterosclerosis}

La isquemia coronaria es considerada la más importante complicación por arterioesclerosis y causa número uno de mortalidad (Molina, 2010; López Pérez J. E., Villar Novell A. L., 2005; Illnait Pérez, 2015; Sevillano, 2013). «Framinghan», relevante estudio sobre el tema, define claramente los principales factores de 
riesgo asociados como: hipertensión arterial e hipertrofia ventricular izquierda, factores como el tabaquismo e intolerancia a la glucosa entre otros (Saínza Rúa et al., 2001). En Colombia las isquemias coronarias son primera causa de mortalidad (Molina, 2010). Según la organización Sanitas Internacional para el 2010, las enfermedades isquémicas del corazón ocuparon en Colombia el primer puesto en cuanto a mortalidad en ambos sexos y en diferentes edades. Si bien una alta proporción de las dislipidemias aterogénicas corresponde a trastornos genéticos, otra proporción también alta se debe a trastornos secundarios a condiciones adquiridas (Ministerio de Salud y Protección Social - Colciencias, 2014; López Pérez, et al., 2005; Romero V, E., y Alemán, R., 2013). Una adecuada prevención y tratamiento en caso de diagnóstico temprano son efectivos para controlar la aparición de enfermedades cardiovasculares (Xavier et al., 2005; Miguel Soca, P. E., 2014; Lértora et al., 2014).

\section{Tratamiento farmacológico}

Se recomienda previo al tratamiento iniciar cambios en la dieta y aumento de actividad física. Las estatinas, también conocidas como inhibidores de la 3-hidroxi-3-metilglutaril coenzima A (HMG-CoA) reductasa, según estudios han mostrado efectos benéficos con una buena respuesta y tolerancia por parte del paciente disminuyendo los valores séricos de pruebas lipídicas, por ende, los eventos vasculares y la muerte (Zárate et al., 2012; López M. et al, 2013).

Los fibratos (derivados del ácido fíbrico) fenofibrato, bezafibrato, ciprofibrato y gemfibrozilo, también han mostrado eficacia en la reducción, sobretodo, en concentración de triglicéridos, debido que aumentan la actividad de la lipoproteína lipasa y reducen la síntesis del Colesterol
(López M. et al., 2013). Existen más fármacos como la ezetimiba, usado como adyuvante al tratamiento con estatinas; esta potencialización permite una reducción en la absorción y liberación del colesterol. Adicional a este, la niacina disminuye la liberación de las lipoproteínas complementando la reducción de los riesgos en los pacientes (López M. et al., 2013).

El objetivo final del tratamiento de la dislipidemia no es solamente la reducción de las concentraciones de lípidos, sino mejorar el tiempo y calidad de vida del paciente por medio de la reducción de las complicaciones de eventos coronarios y disminución de la mortalidad (Romero V. E., y Alemán, R., 2013).

\section{Discusión y Conclusiones}

El resultado de los tratamientos diversos ha mostrado ser una alternativa en prevención de enfermedades coronarias y disminución en presentación de arterioesclerosis. Es importante, sin embargo, acompañar cada tratamiento con cambios de estilo de vida y de dieta en general (Molina, 2010; Núñez Cortés et al., 2011).

Los tres fármacos más usados y presentes dentro de medicamentos esenciales para manejo de dislipidemias en Colombia son estatinas, fibratos y resinas fijadoras; estos han reducido la frecuencia de eventos coronarios en el mundo. Por otro lado, y de acuerdo con resultados de estudios como el realizado por Machado J. et al. (2008), se ha observado que evidentemente hay un mayor efecto en la combinación de los hipolipemiantes. La combinación más acertada es la conformada por lovastatina más gemfibrozilo (Whayne y Thomas, 2007; Machado et al., 2008). En gene- 
ral, el uso esporádico y las terapias aplicadas a individuos con o sin eventos coronarios, pero con algún tipo de predisposición, pueden ser benéficos al mejorar la condición del individuo, y que en caso de hipertrigliceridemia grave, ante el elevado riesgo cardiovascular global o fallo del tratamiento conservador, puede iniciarse tratamiento con varios fármacos para controlar la dislipidemia y reducir el riesgo cardiovascular: fibratos, AGn-3, estatinas, niacina y tiazolidinadionas (Romero V. E., y Alemán, R. (2013); Hooper et al., 2004).

\section{Enfermedad coronaria}

De acuerdo con López (2013), para individuos con dislipidemias, existe una mayor probabilidad de sufrir un evento coronario, especialmente con un inadecuado estilo de vida y si los hábitos alimentarios y el sedentarismo son deficientes. Para algunos es importante el establecimiento de las lipoproteínas de baja densidad (LDL), como estándar de los factores de riesgo CV, (González-Chávez et al., 2011). Otros autores se enfocan hacia protocolos y tratamientos en la búsqueda de disminuir la incidencia de presentación de este tipo de eventos «Scandinavian Simvastatin Survival Study (4S)». Ellos sugieren la simvastatina para reducir el riesgo de desarrollo de insuficiencia cardiaca. Similares estudios como el CARE (Colesterol And Recurrent Events) y LIPID (Long Term Intervention with Pravastatin in Ischaemic Disease) sugieren pravastatina como reductor de insuficiencia cardiaca (Xavier et al., 2005; Laris et al., 2005). Finalmente, el NCEP ATP III recomienda reducir y mantener niveles de LDL-c plasmático a valores menores de 100 mg/dL (Romero V. E., y Alemán, R., 2013; Molina, 2010). También se usa en el mundo la rosuvastatina, medicamento recomendado por la Food and Drug Administration (FDA) (Agencia de Alimentos y Medicamentos o Agencia de Drogas y Alimentos) de los EE.UU. como terapia adicional a la dieta, ya que se observó que puede retardar la generación o en su defecto la progresión de la aterosclerosis en pacientes con hipercolesterolemia (Kryziak R., 2011).

Los fibratos, efectivos por disminuir la grasa que circula por las arterias, participan como reguladores lipídicos disminuyendo los niveles séricos del perfil lipídico, obviamente aumentando el cHDL (Ros, E. y Laguna J. C., 2006) de todas maneras, aunque es efectivo, no se considera como factor (Machado et al., 2008). Adicionalmente, el 4S (Scandinavian Simvastatin Survival Study Group 1994), estudio relevante para estos tratamientos, demuestra que un tratamiento hipocolesterolémico prolongado reduce la morbilidad y mortalidad en la presentación de eventos coronarios.

Revisiones de gran tamaño (Hooper et al., 2004) han permitido determinar las cantidades necesarias para obtener efectos benéficos y satisfactorios, al fijar dosis efectivas para los pacientes.

\section{Hipertensión arterial y diabetes}

A partir de los diferentes estudios realizados, mediante el uso de fármacos a elección, especialmente las estatinas, se logra mejorar considerablemente la condición de los pacientes con hipertensión (Chávez Pardo, et al., 2013). Las estatinas en este caso utilizan un mecanismo que afecta la función endotelial independiente de su capacidad hipolipemiante, porque tienen un efecto sobre el control de la presión arterial. Adicionalmente, el uso de los Omega-3 logra disminuir la aparición de nuevos efectos cardiovasculares como factor protector, ya que si no pueden ser sintetizados 
por el organismo, al ser ingeridos mejoran la circulación (Núñez Cortés et al., 2011) (Fuster y Ruiz 0, 2015).

En el caso de la diabetes, como se dijo anteriormente, el uso de las estatinas (atorvastatina, rosuvastatina, simvastatina y pitavastatina), reduce considerablemente la generación de eventos coronarios y una posterior muerte (Acevedo Rueda, S. M., y Aguilló Prada, R. A., 2004) (Fuster y Ruiz o, 2015) (Ruiz, A. J., 2009). Con respecto a los fenobibratos, su efecto se encuentra relacionado a activación de las citoquinas, células importantes del sistema inmune; dicho efecto mejora la sensibilidad a la insulina y la hemostasia en pacientes diabéticos (Libby P. y Crea F., 2010). Sin embargo, como se ha observado que las combinaciones resultan eficaces es mejor una mezcla de las terapias combinadas aceptadas. (Acevedo Rueda, S. M., y Aguilló Prada, R. A, 2004).

Los diferentes estudios coinciden en que las dislipidemias aumentan el riesgo de aterosclerosis y con ello los factores de riesgo de eventos coronarios en la población latinoamericana mayor de 18 años, por lo tanto y como prevención se ha hecho énfasis en que, adicional a los tratamientos, los pacientes deben hacer cambios drásticos de estilos de vida.

Generalmente, en pacientes con dislipidemias sin otros factores de riesgo asociados se usa un solo hipolipemiante que usualmente es una estatina, aunque en caso de comorbilidades, se ha sugerido combinaciones de fármacos utilizadas en estudios previos y que han demostrado reducir eventos coronarios y mortalidad.

Según los estudios encontrados, se concluye que las estatinas son medicamentos con una excelente respuesta tanto en el tratamiento de la hipertensión como en eventos coronarios y diabetes tipo 2. Con respecto a la hipertensión, se han evidenciado efectos moderado sobre el endotelio capilar corrigiendo su disfunción y con ello una disminución en los valores de la presión arterial.

Ante los eventos coronarios, tras la administración prolongada de estatinas, más allá de lograr el valor meta en el colesterol LDL, se encontró y coincidió en una mejoría en la respuesta coronaria a la acetilcolina, un marcador de la función endotelial, así como una disminución del número de ataques isquémicos.

La recomendación dada por NCEP-ATPIII y apoyada por el estudio VA-HIT es de gran importancia, ya que muestra la existencia de fuertes beneficios con el uso de gemfibrozilo para el tratamiento farmacológico del cHDL en eventos coronarios o en individuos con predisposición y/o riesgo coronario.

\section{Referencias}

- Acevedo Rueda, S. M., y Aguilló Prada, R. A. (2004). Manejo de la dislipidemia en el paciente diabético tipo 2. Medunab, 7(19), 35-40.

- Álvarez de Sotomayor, M., Herrera, M. D., Pérez-Guerrero, C. y Marhuenda, E. (1999). Interés terapéutico de las estatinas en el tratamiento de la ateriosclerosis. Ars Pharmaceutica, 40(4), 217-231.

- Canalizo-Miranda, E., Favela-Pérez, E. A., Salas-Anaya, J. A., Gómez-Díaz, R., JaraEspino, R., Torres-Arreola, L. P., y VlniegraOsorio, A. (2013). Guía de práctica clínica Diagnóstico y tratamiento de las dislipide- 
mias. (Spanish). Revista Médica Del IMSS, 51(6), 700-709.

- Chávez Pardo I, Aguilar Rodríguez M., Rodríguez Pargas A., Cuellar Torres O L (2013). Colesterol, triglicéridos y otros factores de riesgo en pacientes con edema macular diabético. AMC, 7(6), 121-128.

Davies, V. (2007). El 'colesterol bueno' también tiene su lado oscuro contra el corazón. El Nacional (Venezuela). p. 1.

- De los reyes, A. (2013). Obesidad en Latinoamérica, factores detrás del incremento. Euromonitor Internacional. Recuperado de www.tecnomercadoretail.cl/ obesidad-en-latinoamerica-factores

- Fernández Travieso, J. C. (2008a). Manejo de la dislipidemia diabética. Revista CENIC Ciencias Biológicas, 39(1), 16-26.

- Fernández Travieso, J. C. (2008b). Consideraciones genéticas sobre las dislipidemias y la aterosclerosis. Revista CENIC Ciencias Biológicas, 39(3), 161-172.

- Ferrer J. I. (1999). Farmacoterapia de la dislipidemia en el anciano. Revista Cubana Medicina General Integral, 15(4), 478-485.

- Fuster, V. P., Ruiz, E, Pintó Sala, X. (2015). Control de la dislipidemia aterogénica en paciente diabético tipo 2. Clínica e investigación en arteriosclerosis, 27(2), 45-56. doi: 10.1016/j.arteri.2014.06.002

- González-Chávez, A., Simental-Mendía, L. E., y Elizondo-Argueta, S. (2011). Relación triglicéridos/colesterol-HDL elevada y resistencia a la insulina. Cirujía y Cirujanos, 79(2), 126-131.
Hirsch, S., De la Maza, P., Barrera, G., Gattás, V., Petermann, M. y Bunout, D. (2002). The Chilean Flour Folic Acid Fortification Program Reduces Serum Homocysteine Levels and Masks Vitamin B-12 Deficiency in Elderly People. J. Nutr., 132(2), 289-291.

- Hooper L., Thompson R. L., Harrison R. A., Summerbell C. D., Moore H., Worthington H. V., Durrington P. N., Ness A. R., Capps N. E., Davey Smith G., Riemersma R. A., y Ebrahim S. B. (2004). Omega 3 fatty acids for prevention and treatment of cardiovascular disease. Cochrane Database Syst Rev, (4). doi: 10.1002/14651858.CD003177.pub2

- Illnait Pérez, J. (1997). La dislipidemia en el paciente diabético: Parte II Manejo de la dislipidemia en el paciente diabético. Revista Cubana Medicina General Integral. 13(5), 504-511.

Kryziak, R., Gdula-Dymek, A., y Okopien, B.(2011). Simvastatina $Y$ fenofibrato disminuyen la liberación de citoquinas y la inflamación sistémica. American journal of Cardiology, 107(7), 1010-1018.

- Laris, M., Arteaga L., Cuevas, A., y Rigotti, A. (2005). HDL cholesterol: A new target in the treatment of lipid disorders and atherosclerosis? Revista médica de Chile, 133(7), 823-832.

- Lértora, W. J., Villordo, G. I., Mussart, N. B., Catuogno, M. S., y Sánchez Negrette, M. (2014). Desarrollo inicial de ateroesclerosis en aorta de conejos sometidos a dietas con exceso de colesterol. Revista Veterinaria, 25(2), 87-94.

Libby P. y Crea F. (2010). Clinical implications of inflammation for cardiovascular 
primary prevention. European Heart Journal, 31,777-783. doi:10.1093/eurheartj/ehq022 López M. et al. (2013). Guía de Tratamiento Farmacológico de Dislipidemias para el primer nivel de atención. Revista mexicana de cardiología, 24(S1), S23-S37.

- López Pérez J. E., Villar Novell A. L. (2005). Dislipidemias en personas mayores de 60 años. Rev Cubana Med Gen Integr, 21(3-4). Recuperado de http://scielo. sld.cu/scielo.php?script=sci_arttext\&pi$d=S 0864-21252005000300004 \&$ Ing=es

Luengo Fernández, E., Ordóñez Rubio, B., Bergua Martínez, C., Laclaustra Gimeno, M. (2005). Obesidad, dislipemia y síndrome metabólico. Revista Española de cardiología, 5(4), 21-29.

- Machado, J. E., Moncada, J. C., y Mesa, G. (2008). Prescription patterns for antilipidemic drugs in a group of Colombian patients. Revista Panamericana de Salud Pública, 23(3), 179-187.

Machado-Alba, J. E. y Machado-Duque, M. E. (2013). Prevalencia de factores de riesgo cardiovascular en pacientes con dislipidemia afiliados al sistema de salud en Colombia. Revista Peruana de Medicina Experimental $Y$ Salud Publica, 30(2), 205-211.

- Manrique, M., De la Maza M. P., Carrasco, F., Moreno, M., Albala, C., García, J., Díaz, J. y Liberman, C. (2009). Diagnóstico, evaluación y tratamiento no farmacológico del paciente con sobrepeso u obesidad. Rev. méd. Chile, 137(7) 963-971. http://dx.doi. org/10.4067/S0034-98872009000700016

Martínez Hernández, A. F., y Chávez Aguirre, R. (2007). Prevalencia y comorbilidad de dislipidemias en el primer nivel de atención. Revista Médica Del IMSS, 45(5), 469-475.

- Miguel Soca, P. E. (2009). Dislipidemias. Acimed 20(6), 265-273.

- Miguel Soca, P. E. (2014). Marcadores genéticos en la enfermedad cardiovascular. Archivos De Cardiología De México, 84(1), 53-54. doi:10.1016/j.acmx.2013.08.004

- Ministerio de Salud y Protección Social y Colciencias. (2014). Guía de práctica clínica para la prevención, detección temprana, diagnóstico, tratamiento y seguimiento de las dislipidemias en la población mayor de 18 años. Guía para profesionales de la salud. Guía No. 27. Recuperado de http://gpc.minsalud.gov. co/guias/Documents/Dislipidemia/GPC\%20 \%20Dislipidemias\%20profesionales.pdf

Molina, P. A. (2010). Manejo poblacional de las dislipidemias primarias. Revista Médica Clínica Las Condes, 21(5), 705-713.

- Morejón Reinoso, O. y Triana, M. E. (2015). Importancia de la interpretación del colesterol total y de los triglicéridos para el diagnóstico de las dislipidemias. Revista Cubana De Angiología y Cirugía Vascular, (1), 54.

- Romero V. E., y Alemán, R. (2013). Las dislipidemias, el riesgo cardiovascular y su atenuación con rosuvastatina. Informe Médico, 15(4), 175-179.

Ros, E. y Laguna, J. C. (2006). Tratamiento de la hipertrigliceridemia: fibratos frente a ácidos grasos omega-3. Revista española de cardiología, 6(D), 52-61. doi: $10.1157 / 13091599$

Rubiés-Prat J. y Botet J. (2003). Hipertrigliceridemia como factor de riesgo cardio- 
vascular. ¿Fin de la controversia? Medicina Clínica (Barcelona), 120(8), 303-307.

- Ruiz, A. J. (2009). Dyslipidemias and cardiovascular risk: time for a new approach to lipid disorders? Revista Colombiana de Cardiología, 16(5), 214-220.

- Saínza Rúa T., Hernanz Hernanz P., Ortega Inclán N., Mugarza Hernández M. D. (2001). Arteriosclerosis precoz en un paciente con escasos factores de riesgo cardiovascular. Medifam, 11(10), 83-84.

- Sanhueza, J., Valenzuela, R., y Valenzuela, A. (2012). El metabolismo del colesterol: cada vez más complejo. Grasas y Aceites, 63(4), 373-382. doi:10.3989/gya.035512

- Sevillano, A. M., Hernández, E., Caro, J., Molina, M., Gutiérrez, E., Morales, E., y Praga, M. (2012). Ateroembolia de coles- terol y tratamiento combinado con esteroides e iloprost. Nefrologia, 32(6), 824-828. doi:10.3265/Nefrologia.pre2012.Aug. 11645

- Troyo Barriga, P. (2004). Obesidad y dislipidemias. Gac Méd Méx, 140(2), 49-58.

Xavier, H. T., Castellanos, R., y FernándezBritto, J. E. (2005). La asociación de dislipidemia y trombosis en la inestabilización de la placa aterosclerótica. Revista Cubana de Investigaciones Biomédicas, 24(3), 43-47.

Whayne, J. R., Thomas F. (2007). Tratamiento agresivo hipolipemiante en las enfermedades cardiovasculares. Rev. Costarric. Cardiol, 9(2), 11-20.

- Zárate, A., Basurto, L., y Saucedo, R. (2012). El tratamiento del colesterol alterado. Revista Médica Del IMSS, 50(1), 1-4. 